\title{
An Unusual Case of Large-Vessel Vasculitis
}

\author{
Omar Suhail Alsaed, Yousef M. Yahia, Hani Ali Malallah Abdulaziz, Abdul-Wahab Al-Allaf \\ Department of Medicine (Rheumatology), Hamad Medical Corporation, Doha, Qatar
}

Received: $12 / 04 / 2018$

Accepted: 20/04/2018

Published: $17 / 05 / 2018$

How to cite this article: Alsaed OS, Yahia YM, Abdulaziz HAM, Al-Allaf AW. An unusual case of large-vessel vasculitis. EJCRIM 2018;5: doi:10.12890/2018_000897.

Conflicts of Interests: The Authors declare that there are no competing interests.

This article is licensed under a Commons Attribution Non-Commercial 4.0 License

\section{ABSTRACT}

We report the case of a previously healthy 35-year-old man who presented with severe abdominal pain, nausea, vomiting and subjective fever and was found to have acute kidney injury, haematuria, leukocytosis and elevated inflammatory markers. An abdominal CT scan showed lobar nephronia of the left kidney complicated by infarction. Subsequent MRI also revealed splenic infarction. Despite IV antibiotics and US-guided perinephric collection drainage, the condition of the patient continued to deteriorate so he underwent total nephrectomy. Serial follow-up CT scans showed multi-level vascular occlusions, bowel ischaemia and splenic infarction. Large-vessel vasculitis was suspected, and pulse steroid therapy was planned. However, the histopathology report of the resected kidney revealed mucor-like fungal infection suggestive of invasive mucormycosis as a cause for the widespread vasculitis. Although IV amphotericin B and caspofungin were started immediately, the patient died a few days later. We report this case to raise awareness that invasive fungal infection can cause largevessel vasculitis. Immunosuppression for patients from endemic areas should only be considered after an infectious aetiology for vasculitis has been excluded.

\section{LEARNING POINTS}

- Although the cause of vasculitis is not usually known, infectious agents can be implicated.

- Infectious causes should always be considered in patients from endemic areas.

- We report mucormycosis as a cause of widespread large-vessel vasculitis.

\section{KEYWORDS}

Vasculitis, Infection, Mucormycosis

\section{INTRODUCTION}

Vasculitides are inflammatory conditions that involve vessel walls. The affected vessels vary in size and location. Vasculitis can be a primary autoimmune disorder or secondary to other diseases or drugs. Differentiation between primary and secondary types of vasculitis is usually challenging and needs comprehensive review of the patient's clinical presentation, background, blood tests and imaging findings. Many infections such as syphilis (a well-known cause of aortitis) can cause vasculitis. HBV, HCV and CMV infection may result in viral-associated vasculitis ${ }^{[1]}$, while fungal infection can also cause vasculitis ${ }^{[2]}$. This is important for patients from endemic areas. Identification of the infectious causes of vasculitis and avoidance of immunosuppression are vital for disease prognosis. We report a case of medium/large-vessel vasculitis secondary to a very unusual infection in an immunocompetent patient. 


\section{CASE DESCRIPTION}

A 35-year old Indian man with no previous comorbidities presented with severe abdominal pain, nausea, vomiting, constipation, haematuria and subjective fever for a couple of days prior to emergency room admission. The abdominal pain was diffuse and of gradual onset, and was not colicky or radiating to the back. The patient did not report any cardiovascular, chest or musculoskeletal symptoms. There were no previous similar episodes.

Physical examination showed a temperature of $38.1^{\circ} \mathrm{C}$ (oral), respiratory rate 17 , blood pressure $122 / 81$, pulse $95 / \mathrm{min}$ and $\mathrm{SpO} 298 \%$ on room air. The patient had an average physique and was not jaundiced or pale. Focused abdominal examination showed a diffusely distended abdomen with maximum tenderness over the left hypochondrium area. Bowel sounds were normal. The liver and spleen were not enlarged. Cardiovascular and chest examinations were unremarkable.

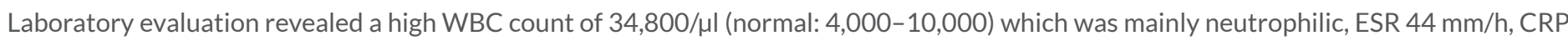
$300 \mathrm{mg} / \mathrm{l}$ (normal: <5), procalcitonin $3.97 \mathrm{ng} / \mathrm{ml}$ (normal: <0.5), creatinine 135 mmol/l (normal: <110), ALT 182 U/l (normal: 5-55), AST 123 U/I (normal: 5-34), ALP $156 \mathrm{U} / \mathrm{I}$ (normal: 40-150) and total bilirubin 126 umol/I (normal: 3.4-20.5). Urine examination showed an elevated WBC of $90 \times 10^{9} /$ (normal: $0-9 \times 10^{9}$ ) and RBC of $26 \mu$ l (normal: $0-5$ ). Several sets of blood and urine cultures were all negative.

The initial impression was obstructive uropathy or intestinal obstruction. An urgent CT scan of the abdomen with contrast revealed a large well-defined, wedge-shaped, mildly swollen, poorly perfused area with minimal patchy enhancement in the left kidney. This was suggestive of lobar nephronia of the upper and mid poles with a possibility of renal infarction or a neoplastic process (Fig. 1A). There was no evidence of intestinal obstruction. The patient underwent urgent MRI for further clarification. This confirmed the enlarged and oedematous left kidney with a wedge-shaped area at the upper and interpolar region. The involved area revealed heterogeneous signalling: predominant high intensity on T2 and diffusion restriction with no significant enhancement in the post-contrast sequences. There was no significant contrast excretion from the left kidney on the delayed post-contrast sequences. This initial MRI showed that the main renal artery and renal veins were patent. This was suggestive of lobar nephronia probably complicated by infarction with no features of angiomyolipoma (Fig. 1B). Despite the administration of a broad-spectrum antibiotic, IV fluids and other symptomatic treatment, the patient's abdominal pain continued to worsen, fever persisted, and there was a steady increase in serum WBC, inflammatory markers and serum creatinine. Three days later, the patient underwent ultrasonography which revealed significantly reduced perfusion of the left kidney compared with the right kidney with a small left perinephric collection at the interpolar region measuring $2.5 \times 0.6 \mathrm{~cm}$. US-guided drainage of the perinephric collection removed $10 \mathrm{ml}$ of fluid, which was negative for Gram stain and culture and negative for TB culture. However, as the patient did not improve after drainage, total left side nephrectomy was performed to eliminate the source of infection. A post-operative CT scan of the abdomen with contrast revealed total occlusion of the proximal part of the splenic artery, right renal artery, accessory right renal artery and lower half of the superior mesentery vein, and a thickened bowel wall suggestive of ischaemia (Fig. 2A).

At this stage, the patient was referred to the rheumatology service as a case of large-vessel vasculitis based on left renal infarction, splenic infarction, bowel ischaemia and multi-level vessel occlusions (Fig. 2).

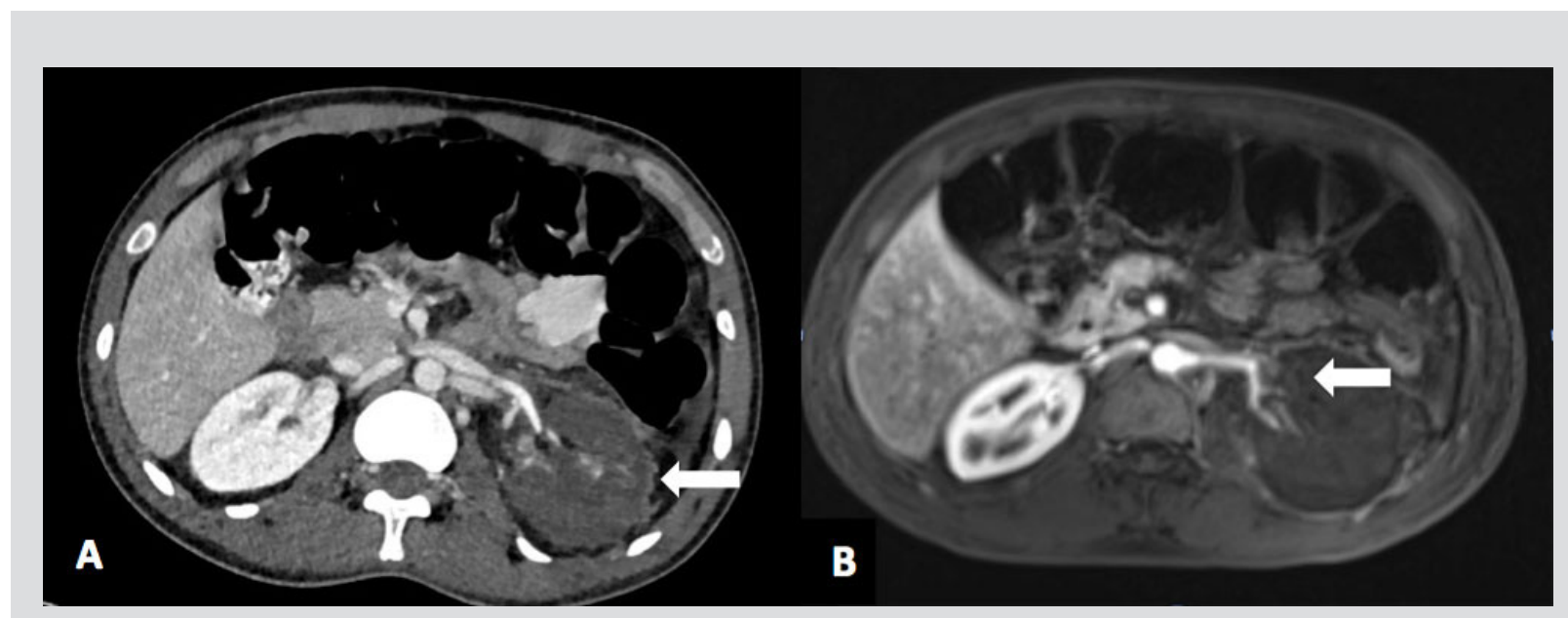

Figure 1. (A) CT scan of the abdomen with contrast showing left lobar nephronia with possible infarction or a neoplastic process. (B) MRI (T1 post-contrast) demonstrating heterogenous signalling with patent renal vasculature 

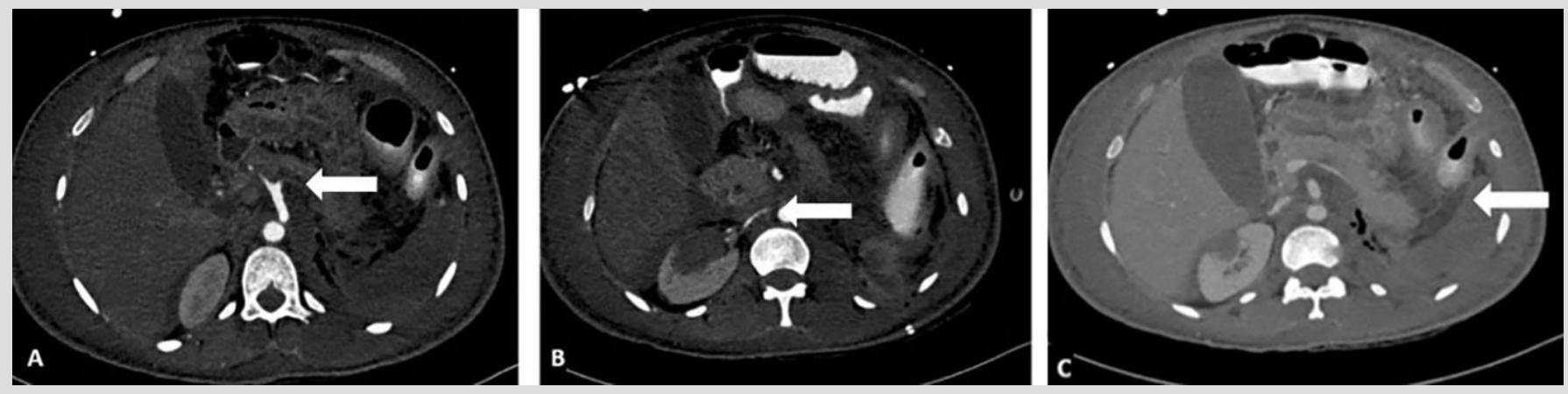

Figure 2. CT scan of the abdomen with contrast: (A) splenic artery occlusion; (B) right renal artery occlusion with renal infarction; (C) splenic infarction

However, before administering immunosuppressive therapy to the patient, the rheumatology team reviewed the resected kidney with the pathologist, looking for findings suggestive of autoimmune vasculitis rather than a thrombotic event. Surprisingly, the resected kidney showed extensive mucor-like fungal infection causing dense vessel wall inflammation with infarcted necrotic tissue. All autoimmune immunology profiles investigating ANA, Ds DNA, ANCA, antiphospholipid syndrome antibodies (lupus anticoagulant, B2 glycoprotein Ab, anticardiolipin $\mathrm{Ab}$ ) and thrombophilia were unremarkable. The presence of invasive mucormycosis is very unusual in an immunocompetent patient (our patient had shown negative serology for HIV, HBV and HCV). Peripheral smears did not reveal any blast cells. Pan CT scans showed partial opacification of the right maxillary air sinus but were negative for hidden malignancies.

Despite receiving broad-spectrum antibiotics, dual antifungal therapy (amphotericin B and caspofungin), heparin infusions and multidisciplinary care from different specialties (SICU, urology, nephrology, infectious disease, haematology and rheumatology) the patient developed multi-organ failure with DIC, renal failure and bilateral pleural effusion with ARDS, and died 19 days after admission.

\section{DISCUSSION}

Disseminated mucormycosis (also known zygomycosis) is a devastating fungal infection with a very high mortality rate of $90-100 \%{ }^{[3]}$. The most common primary infections are rhino-orbital-cerebral and pulmonary mucormycosis, followed by cutaneous, cerebral, disseminated and renal zygomycosis. The finding of invasive mucormycosis in an immunocompetent individual, such as our patient, is uncommon. In one large case series of mucormycosis (1,999 patients) from India, 22.9\% of subjects were healthy ${ }^{[4]}$. In another case series from Europe, no underlying condition could be identified in $8 \%$ of the cohort ${ }^{[5]}$. Secondary vasculitis and mycotic thromboembolism are rare complications of the disseminating form of mucormycosis. Vasculitis results from direct invasion by fungal filaments of the vascular wall with consequent inflammatory activation. This has been reported in small-vessel vasculitis ${ }^{[2]}$. Our patient developed both large and small-vessel vasculitis, with fungal filament invasion of the large vessels causing ischaemic changes in the bowel, splenic infarction and left kidney infarction. This was proven by the initial CT scan with contrast which showed these changes without evidence of thrombotic events at that stage. However, a few days later, imaging scans revealed multi-level vascular occlusions which suggested thromboembolic events. Inflammatory cells were seen in the vessel walls in the resected kidney. As far as we know, large/medium-vessel vasculitis induced by mucormycosis has rarely been reported.

Identification of the infectious aetiologies of secondary vasculitis is critical for determining the therapeutic strategy for the patient. Usually, antibiotics, antiviral or antifungal agents are used to treat secondary infectious vasculitis, depending on the causative organism, and immunosuppression is rarely needed. A high index of suspicion early in the course of the disease to exclude the possibility of an infectious cause in patients from endemic areas, is very important for a successful outcome.

In conclusion, physicians should have a high index of suspicion for secondary causes of vasculitis before administering immunosuppressive therapy. 


\section{REFERENCES}

1. Pagnoux C, Cohen P, Guillevin L. Vasculitides secondary to infections. Clin Exp Rheumatol 2006;24(2 Suppl 41):S71-S81.

2. Royer M, Cervera P, Kahan A, Menkès C-J, Puéchal X. Mucormycosis cerebral arteritis mimicking a flare in ANCA-associated vasculitis. Lancet Infect Dis 2013;13:182.

3. Roden MM, Zaoutis TE, Buchanan WL, Knudsen TA, Sarkisova TA, Schaufele RL, et al. Epidemiology and outcome of zygomycosis: a review of 929 reported cases. Clin Infect Dis 2005;41:634-653.

4. Chakrabarti A, Das A, Sharma A, Panda N, Das S, Gupta KL, et al. Ten years' experience in zygomycosis at a tertiary care centre in India. J Infect 2001;42:261-266.

5. Skiada A, Pagano L, Groll A, Zimmerli S, Dupont B, Lagrou K, et al. Zygomycosis in Europe: analysis of 230 cases accrued by the registry of the European Confederation of Medical Mycology (ECMM) Working Group on Zygomycosis between 2005 and 2007. Clin Microbiol Infect 2011;17:1859-1867. 